\title{
ESTRUCTURA SOCIOESPACIAL DE LA AGLOMERACIÓN GRAN BUENOS AIRES ${ }^{1}$
}

\section{ESTRUTURA SOCIOESPACIAL DA AGLOMERAÇÃO GRANDE BUENOS AIRES}

\author{
Mariana Marcos \\ Facultad de Ciencias Sociales de la Universidad de Buenos Aires, Buenos Aires, Argentina, \\ mariana_marcos_00@yahoo.com.ar
}

\begin{abstract}
RESUMEN
El presente artículo se inscribe en la intersección de dos líneas de investigación con larga trayectoria en la Argentina: los estudios abocados a la estructura social de la población en sentido amplio, abarcando la identificación de grupos sociales que se diferencian por su posición en la estructura productiva, sus comportamientos demográficos y sus condiciones de vida; y los trabajos que espacializan la problemática de las desigualdades socioeconómicas intraurbanas. En este marco, se pretende conocer cómo se configuran espacios diferenciales a nivel intra-metropolitano en Buenos Aires, a partir de la condición socioeconómica y las estructuras y dinámicas demográficas de la población. El diseño metodológico de tipo cuantitativo, de escala microespacial y perspectiva exploratoria. Los resultados se analizan a la luz de los antecedentes acerca de las relaciones socioespaciales pasadas y presentes de producción y reproducción del espacio metropolitano.
\end{abstract}

Palabras clave: Buenos Aires. Distribución espacial de la población. Geo-demografía.

\section{RESUMO}

O presente artigo inscreve-se na interseção de duas linhas de investigação com longa trajetória na Argentina: os estudos dirigidos à estrutura social da população em sentido amplo, tomando em conta a identificação de grupos sociais que se diferenciam por sua posição na estrutura produtiva; seus comportamentos demográficos e suas condições de vida; e os trabalhos que estudam a problemática das desigualdades socioeconômicas ó no interior do espaço urbano. Neste marco, pretende-se conhecer como se configuram espaços diferenciais a nível intrametropolitano em Buenos Aires, a partir da condição socioeconômica e as estruturas e dinâmicas demográficas da população. O desenho metodológico é de tipo quantitativo, de escala microespacial e de caráter exploratório. Os resultados analisam-se à luz dos antecedentes acerca das relações socioespaciais passadas e presentes de produção e reprodução do espaço metropolitano.

Palavras-chave: Buenos Aires. Distribuição espacial da população. Geo-demografía.

Artigo recebido para publicação em janeiro de 2015

Artigo aceito para publicação em maio de 2015

\section{INTRODUCCIÓN}

La preocupación por conocer las características y la dinámica que adquiere la distribución espacial de los grupos sociales en las ciudades tiene una larga trayectoria en las ciencias sociales del último siglo: desde lo que se reconoce como el inicio de los modernos estudios urbanos, con los trabajos

\footnotetext{
1 Este artículo presenta los resultados de la investigación "Más allá de los promedios: diferencias y especificidades intraurbanas. La estructura demo-espacial de la Aglomeración Gran buenos Aires en el año 2001”. Se agradecen los valiosos comentarios y recomendaciones de los directores, Susana Torrado y Guillermo Velázquez, y de los colegas amigos Gustavo Buzai, Antonio Moreno Jiménez y Gabriela Mera, y la traducción al portugués de Mónica Bankirer. Los errores que pudiera haber son responsabilidad de la autora.
} 
desarrollados en la Universidad de Chicago a comienzos del siglo XX -encabezados por Robert Park, Ernest Burgees y Roderick McKenzie- hasta los aportes de la llamada nueva sociología urbana -con Henri Lefebvre, Manuel Castells y David Harvey, entre otros-, que vinieron a problematizar la espacialidad, poniéndola en relación con la dinámica de producción, reproducción y cambio social. Lo que se plantea desde esta línea es rescatar al espacio como una dimensión de análisis fundamental para comprender los procesos sociales, entendiendo que éste es producto y productor de las relaciones y prácticas que lo atraviesan (Lefebvre, 1972; Soja, 1989; Harvey, 2000).

En este marco, un importante corpus de trabajos académicos ha procurado dar cuenta de la distribución espacial de los grupos sociales en las grandes ciudades latinoamericanas y de los cambios de considerable magnitud que ha experimentado desde las últimas décadas del siglo XX, con la emergencia de elementos totalmente novedosos, la transformación y resignificación de otros prexistentes que cobrarían protagonismo, el pasaje a segundo plano o la desaparición de elementos que antes fueron relevantes, y la permanencia de formas socioespaciales propias del particular ordenamiento socioterritorial anterior.

El presente trabajo se centra en Buenos Aires, y se propone indagar en la configuración de espacios diferenciales a nivel intra-metropolitano, a partir de la condición socioeconómica y las estructuras y dinámicas demográficas de la población. El objetivo que aquí se persigue tiene anclaje en la intersección de dos líneas de investigación con larga trayectoria en la Argentina: los estudios abocados a la estructura social de la población en sentido amplio, abarcando la identificación de grupos sociales que se diferencian por su posición en la estructura productiva, sus comportamientos demográficos y sus condiciones de vida; y los trabajos que espacializan la problemática de las desigualdades socioeconómicas intraurbanas.

La preocupación por la estructura social estuvo presente desde los momentos fundacionales de la sociología argentina. Desde el trabajo pionero de Germani y Graciarena (1955) Estructura social de la Argentina. Análisis estadístico, hasta los actuales de Torrado (1992; 1995; 2003; 2010, entre los más importantes) se puede trazar un recorrido en el que, en términos generales, la escala espacial 
se fue afinando progresivamente, partiendo de los estudios a nivel del total del país, para distinguir luego diferenciales regionales, provinciales e inclusive algunas tendencias básicas en el interior de Buenos Aires. Sin embargo, la dimensión espacial nunca ocupó un lugar protagónico, al menos en los trabajos integrales que abordan tanto la estructura ocupacional como las estructuras y dinámicas demográficas de la población.

Con un desfasaje temporal de aproximadamente veinte años, desde el urbanismo y la geografía social cuantitativa comenzaron a realizarse grandes esfuerzos para estudiar la heterogeneidad socioespacial interna de Buenos Aires. Los primeros trabajos de Torres datan de mediados de la década de 1970 (Torres, 1977; 1978) y estudiaban la configuración del espacio metropolitano como producto de la mutua interacción entre el territorio, o medio natural modificado por la acción del hombre, y la organización social de la producción y el consumo (Torres, 1993). Siempre desde esta perspectiva, a lo largo de sus investigaciones el autor exploró la condición socioeconómica y habitacional de la población y el soporte físico infraestructural (Abba, 2011).

Desde mediados de la década del 2000, a partir de la publicación de la primera base de datos censales en formato digital en Argentina, se asiste a una explosión de trabajos sobre Buenos Aires -y sobre muchas otras ciudades del país-, a la vez macrosociales, en tanto abordan a la ciudad en su totalidad, y microespaciales, en la medida que la preocupación es visibilizar las diferencias sociales a nivel de pequeñas unidades espaciales. Los esfuerzos van desde la actualización del mapa social de Horacio Torres a partir de indicadores univariados o multivariados de nivel socioeconómico (Thuiller, 2005; Groisman y Suárez, 2009; Marcos, 2011; Buzai y Marcos, 2012), hasta trabajos más específicos que estudian las características e impacto de estrategias residenciales particulares y propias de grupos sociales determinados -concretamente, las villas miseria y asentamientos, y los barrios cerrados-. Desde los ámbitos de gestión también comienza a publicarse información espacializada, acompañada por análisis básicos, como es el caso del Atlas Ambiental del Buenos Aires (con participación del Gobierno de la Ciudad Autónoma de Buenos Aires y otros organismos e instituciones estatales), o más profundos, como en el trabajo impulsado desde Dirección Provincial de Ordenamiento Urbano 
Territorial de la Provincia de Buenos Aires, Lineamientos Estratégicos para la Región Metropolitana de Buenos Aires (DPOUyT, 2007).

A pesar de estos importantes avances, el abordaje integral y en profundidad de la configuración espacial de las características sociodemográficas de la población en Buenos Aires continúa siendo una cuenta pendiente, en el sentido de que hasta ahora, salvo pocas excepciones, el nivel socioeconómico ha sido definido de forma simple y sin tener en cuenta sus múltiples dimensiones, y la espacialización de las estructuras y fenómenos demográficos es muy incipiente.

La propuesta es aquí, precisamente, llevar adelante dicha tarea, afrontando los importantes desafíos que ella supone. Para tal fin se dispone de los datos del Censo Nacional de Población, Hogares y Viviendas del año 2001, el único Censo argentino que brinda información acerca de todas las estructuras y fenómenos abordados en este estudio a nivel de unidades espaciales pequeñas.

\section{La Aglomeración Gran Buenos Aires}

La Aglomeración Gran Buenos Aires (AGBA) denomina a la envolvente de población o mancha urbana más grande de Argentina, que tiene como núcleo a la Ciudad Autónoma de Buenos Aires (CABA) y se extiende hasta donde tiene continuidad la concentración de edificaciones vinculadas a través de las vías de comunicación (Vapñarsky, 1995; 2000), abarcando en forma total o parcial a otras treinta y dos áreas de gobierno local (Partidos de la Provincia de Buenos Aires) (Figura 1).

En la actualidad, con sus 13.588.171 habitantes (Censo 2010), la AGBA concentra a la tercera parte de la población del país y tiene una consolidada primacía ${ }^{2}$ en el sistema de asentamiento argentino (Vapñarsky, 1995). Tanto el posicionamiento de la ciudad en ese lugar cardinal como sus características internas constituyen la síntesis resultante de un entramado de prácticas que operan en diferentes niveles: lógicas económicas, sociales, políticas y culturales globales; oportunidades

2 Vapñarsky (1995) define a la primacía como el fenómeno por el cual la aglomeración primada (en este caso, la Aglomeración Gran Buenos Aires) excede en más del doble a la siguiente en magnitud de población. 
habitacionales generadas y/o condicionadas por políticas públicas y por la dinámica del mercado inmobiliario; estrategias de los hogares para captar recursos y satisfacer sus necesidades habitacionales; y fronteras de tipo social y simbólico que operan en un plano más relacionado con las representaciones, influyendo también en las expectativas habitacionales y los patrones de asentamiento de los grupos sociales en la ciudad. Si bien no es objetivo de este trabajo dar cuenta de esos procesos, es imprescindible describir -así sea de manera esquemática- algunos de sus rasgos principales, en la medida que la estructura sociodemográfica de la ciudad que se pretende hacer emerger constituye la cristalización de ellos en un momento determinado.

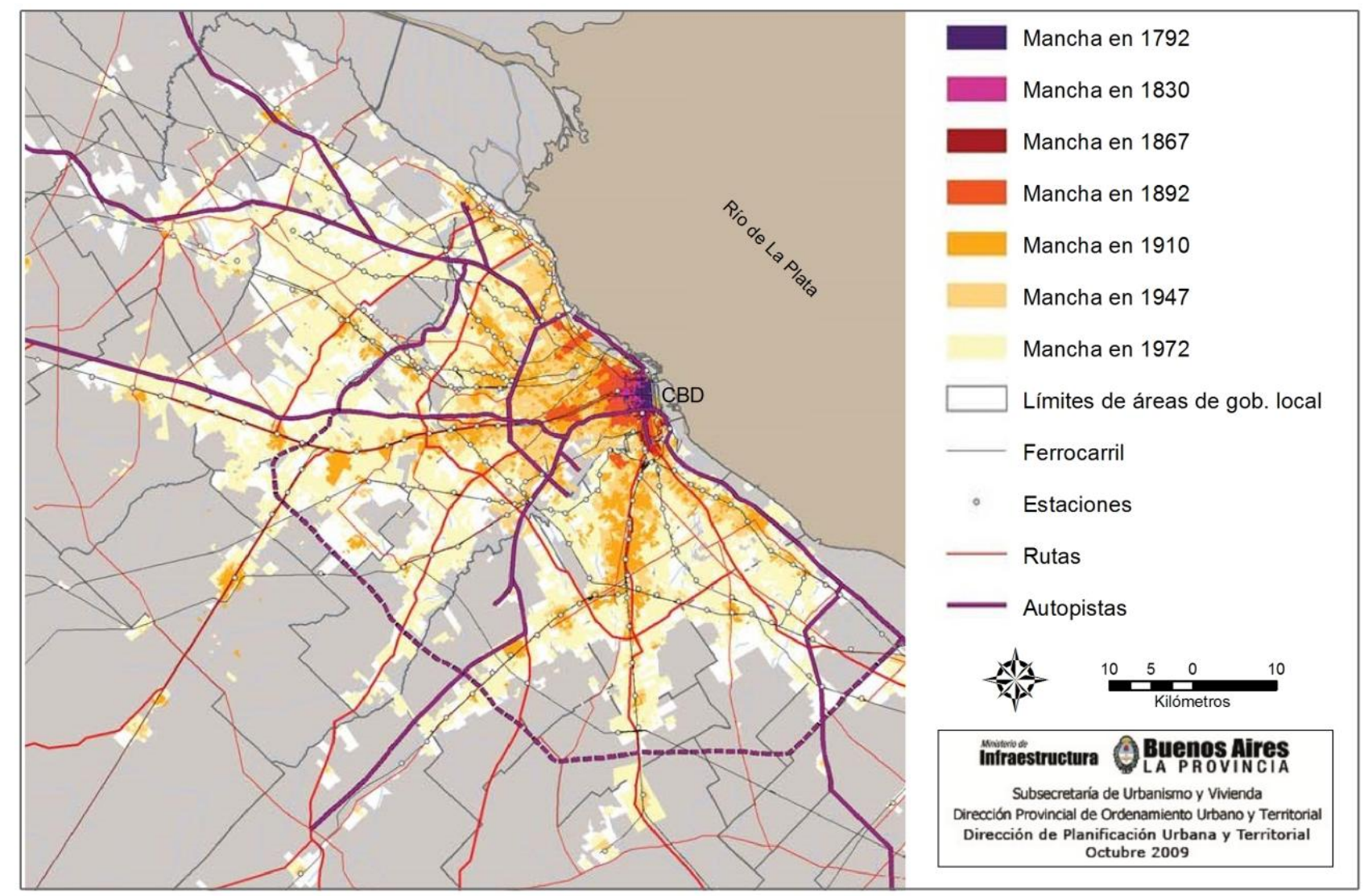

Figura 1. Crecimiento de la Aglomeración Gran Buenos Aires entre 1782 y 2001.

Fuente: modificado de DPOUyT (2007).

Como primera cuestión, cabe señalar que la primacía de Buenos Aires se remonta al período colonial, pero su consolidación se da con posterioridad a la crisis de 1930 , al entrar en vigencia la industrialización por sustitución de importaciones (ISI), que atrajo grandes contingentes poblacionales 
desde el interior del país hacia las aglomeraciones de mayor desarrollo industrial, en especial, hacia la AGBA (Lattes, 2007).

Durante esta etapa, los principales elementos estructurantes de la ciudad fueron los emprendimientos productivos privados de tipo fabril y las políticas públicas en materia de transporte, vivienda, gestión del suelo y promoción industrial.

Atraídas por la disponibilidad de servicios, mano de obra y un gran mercado, las fábricas se instalaron fuera de la CABA, conformando un anillo que la rodea, y operaron, a su vez, como factor de atracción de trabajadores que conformaron áreas residenciales populares en sus inmediaciones (Briano, Fritzsche y Vio, 2003).

A su vez, el Estado nacional realizaba por entonces inversiones e intervenciones directas sobre el territorio. En el proceso de suburbanización de las clases populares tuvo un rol protagónico el subsidio al transporte urbano -fundamentalmente del ferrocarril-, que funcionó como subsidio indirecto a la tierra residencial suburbana, abaratando los costos de los desplazamientos cotidianos de la población económicamente activa entre el lugar de residencia y el lugar de trabajo (Torres, 2001). La expansión y poblamiento de la cuadrícula inicial también fue propiciada desde el Estado mediante los loteos económicos periféricos y la legislación poco restrictiva, que permitieron a los trabajadores acceder a un terreno propio y autoconstruir allí sus viviendas (Prevot Schapira, 2002; Torres, 2001).

Hacia finales de la etapa desarrollista, la AGBA, como otras grandes ciudades industriales latinoamericanas, se caracterizaba por tener un área central concentrada y bien delimitada, con vértice en el centro administrativo y de negocios (central business district-CBD-) y extendida a través de un eje de crecimiento definido (en este caso, hacia el norte de la periferia), en el que se asientan las clases altas en las viviendas de mejor calidad y dotadas de todos los servicios (Janoschka, 2003; Sabatini 2003). 
Fuera del centro, la cuadrícula urbana se extiende de manera continua hasta los límites de la aglomeración, que coinciden con el alcance del ferrocarril. A mayor distancia con respecto al centro, menor es el nivel socioeconómico de la población, la calidad de las viviendas y la dotación de servicios urbanos (pavimento, agua corriente, cloacas, transporte público regular), con la importante excepción de los subcentros de servicios en los Partidos del Conurbano que más habían crecido en las décadas de 1960 y 1970 (Ciccolella, 1999).

Pero a pesar de esta diferenciación socioespacial interna en la ciudad, en buena parte del período desarrollista el crecimiento económico combinado con gobiernos de corte populista hizo de la ciudad un ámbito de integración en el que los barrios tenían cierta diversidad social (Sabatini, 2003). No obstante, los asentamientos precarios, el deterioro de algunas áreas cercanas al centro y, principalmente, los enclaves de pobreza denominados "villas miseria", encarnaron los límites de los mecanismos de integración (Torres, 2001; Prevot Schapira, 2003).

A partir de la década de 1970 -y aproximadamente hasta el año $2003^{3}$-, la Aglomeración comienza a experimentar cambios que modifican sensiblemente tanto su lugar en el sistema de asentamiento nacional como su estructura interna. Por un lado, el atractivo de la principal ciudad del país decayó junto a su capacidad de emplear mano de obra industrial (Vapñarsky, 1995; Rofman, 1999; 2005; Meichtry, 2007), y se inauguró un proceso de redistribución de la población que favoreció a los destinos no-metropolitanos, conocido como proceso de “contraurbanización” o "contraprimacía" (Meichtry, 2007), o "etapa post-expansiva" (Gorelik, 2009). Y, al mismo tiempo, la ciudad compacta, que crecía avanzando sobre el campo abierto en forma de mancha de aceite, dio progresivamente lugar a un espacio urbano de bordes difusos, dotado de múltiples centros, que parece crecer en forma tentacular o de red, y cuyo territorio presenta cada vez más profundas diferencias micro-espaciales, en contraposición con las gruesas diferencias entre espacios de gran tamaño que predominaban durante la etapa desarrollista (Ciccolella, 1999).

\footnotetext{
${ }^{3}$ Autores como García Delgado (2006), Varesi (2010), Basualdo (2011) y Aspiazu et al (2011) coinciden en señalar el año 2003 como el comienzo de un nuevo modelo de acumulación que dan en llamar "Post-convertibilidad", "Neodesarrollismo" o Post-neoliberalismo".
} 
Estas transformaciones emergen en un contexto global y nacional particular, signado por la globalización del régimen de acumulación capitalista en su etapa tardía y la instauración de un modelo de acumulación aperturista en la Argentina. Para la AGBA, ello significó su progresiva integración en una compleja trama de redes productivas transnacionales, con sedes alternativas para la localización estratégica -direccionada por grandes empresas, grupos económicos transnacionales, mercados financieros e instituciones internacionales - de centros de trabajo, inversiones, aprovisionamiento de materias primas e insumos y nuevos mercados (Ciccolella, 1999). En este marco, en la estructura económica metropolitana cobran relevancia y visibilidad determinados actores, pierden incidencia otros, y emergen como dinámicos sectores productivos también particulares, con significativas repercusiones directas e indirectas en la producción del espacio metropolitano.

Entre los actores dinamizadores de este proceso de reconversión económico-estructural y espacial de la principal ciudad del país, se encuentran el capital privado extranjero y los grupos inversores locales, vinculados los primeros a los servicios privatizados, los grandes equipamientos de consumo, ocio y espectáculo, la nueva hotelería internacional, la reconversión industrial y los grandes proyectos urbanísticos, y los segundos a la construcción de urbanizaciones cerradas y sistemas de autopistas (Ciccolella, 1999; 2007). El Estado, antes protagonista de intervenciones directas en matera de planificación, producción y organización del espacio urbano, pasa a ejercer el rol más "económico" de acondicionador y promotor (Briano, Fritzsche y Vio, 2003), y los emprendimientos que modifican el paisaje urbano quedan en manos de quienes tienen los recursos económico-financieros para llevarlos adelante (Janoschka, 2002).

Hacia finales de la etapa aperturista -que precisamente es cuando se observará la estructura sociodemográfica de la ciudad a causa de la disponibilidad de datos- "el aprovechamiento de las ventajas locales para la acumulación" se había erigido como principio rector de los emprendimientos económico-territoriales, el capital privado nacional e internacional ejercía en los hechos el rol de desarrollador urbano y había generado condiciones de posibilidad para novedosos patrones de crecimiento y ordenamiento de la ciudad y asentamiento de la población, y la desarticulación de la 
matriz productiva desarrollista diezmaba el mercado de trabajo, produciendo un proceso de movilidad social estructural descendente (Torrado, 2010) que en la ciudad se puso de manifiesto como "reversión perversa de la segregación" (Sabatini, 2003) por empobrecimiento de las clases medias.

Entre los rasgos de la estructura urbana más sobresalientes, debe mencionarse la especialización territorial estructurada en "islas" dotadas de desarrollos urbanos y artefactos arquitectónicos específicos, y conectadas entre sí por una red ampliada y renovada de autopistas y autovías, y por dispositivos comunicacionales novedosos. Siguiendo la clasificación de Janoschka (2002), se pueden distinguir islas de producción (parques industriales, las sedes empresariales con edificios corporativos y hoteles internacionales de alta categoría), islas de consumo (centros comerciales, de entretenimiento y espectáculos), e islas residenciales de precariedad (villas miseria) y de riqueza (barrios cerrados en las afueras de la aglomeración ${ }^{4}$, edificios de categoría y áreas refuncionalizadas en el centro). Puede tratarse de emprendimientos totalmente nuevos desarrollados y comercializados en forma privada, o en otros casos son viejas estructuras urbanas que han renovado su importancia (por ejemplo, es el caso de las islas de precariedad -o también villas-) o han sido recicladas para nuevos usos. En todos los casos, el denominador común es la "emancipación” de la localización de los emprendimientos con respecto a la red de ferrocarriles, y el reconocimiento del nuevo límite de la suburbanización residencial y productiva en el área de influencia de las autopistas. Las "islas" irrumpen en la estructura urbana preexistente fragmentando su continuidad y la amplían en la periferia sin necesaria continuidad espacial, y desafiando, en consecuencia, la definición misma de aglomerado que utiliza el sistema estadístico argentino.

Los datos que se utilizaron en esta investigación corresponden precisamente al final de esta etapa aperturista, cuando sus elementos metropolitanos característicos ya se superponían y articulaban sobre las capas de espacio desarrollista preexistentes.

\section{METODOLOGÍA}

\footnotetext{
${ }^{4}$ La localización de las villas, asentamientos y barrios cerrados puede consultarse en la cartografía de DPOUyT, 2007.
} 
En el diseño metodológico es de tipo cuantitativo, combinándose a la vez la escala macrosocial, puesto que se analiza a la ciudad en su conjunto, y la microespacial, dado que las unidades de análisis son las unidades espaciales pequeñas que se distinguen en el interior de la AGBA. El carácter exploratoriodescriptivo del abordaje permite arribar de forma inductiva a espacios intrametropolitanos diferenciales -definidos por las características socioeconómicas y sociodemográficas de la población que se asienta en ellos-, que se analizan a la luz de los antecedentes bibliográficos acerca de las relaciones socioespaciales pasadas y presentes de producción y reproducción social.

La fuente de datos principal es el Censo Nacional de Población, Hogares y Viviendas 2001 (INDEC). La información censal alfanumérica se asocia a una cobertura cartográfica digital de unidades espaciales pequeñas especialmente construida para este estudio en base a la cartografía censal original $^{5}$. Desde una perspectiva macrosocial cuantitativa y a la vez microespacial como la que se ha propuesto, la información censal -al ser relevada en forma universal- provee a la vez una flexibilidad óptima y única "para análisis a niveles microespaciales, asegurando representación para cualquier definición de unidades. Por el contrario, la generada por muestreo sólo tendrá representatividad confiable a niveles de agregación particulares y/o para unidades espaciales predefinidas, determinados éstos por el método de muestreo utilizado" (Robirosa, 1996: 629). En este sentido, el Censo de Población es la fuente por antonomasia para hacer este tipo de estudios. Sin embargo, en Argentina el Censo de 2001 es el único que cumple a la vez con los requisitos de poner a disposición del público una base de datos usuario y de haber recolectado la totalidad de la información de forma universal, sin aplicar procedimientos de muestreo (como es el caso del más reciente Censo 2010); es decir que es el

\footnotetext{
${ }^{5}$ La metodología que se siguió para construir la cartografía de este estudio puede consultarse en Marcos, 2011.
} 
único Censo que brinda información acerca de todas ${ }^{6}$ las estructuras y fenómenos abordados en este trabajo a nivel de unidades espaciales pequeñas?

La utilización del Censo como fuente de datos principal complejiza y dificulta el abordaje de algunos fenómenos demográficos, que requeriría de las estadísticas vitales, cuyas posibilidades de desagregación espacial son sumamente reducidas. En esos casos, se recurre a técnicas indirectas de estimación demográfica que permiten calcular indicadores básicos a partir de datos censales. Dichas medidas - pensadas originalmente para paliar la falta de estadísticas vitales de calidad en los países no desarrollados- se utilizan aquí como alternativa ante estadísticas vitales de calidad, pero con escasa flexibilidad para brindar la información requerida para estudios microespaciales.

La información censal se analiza mediante la técnica de análisis factorial de componentes principales en su variante exploratoria. El análisis factorial -muy utilizado en los estudios microespaciales cuantitativos para facilitar la manipulación de grandes volúmenes de información- busca sintetizar la información de las variables originales en un número mínimo e imprescindible de nuevas variables denominadas factores (Santos Preciado, 1991; Buzai, 2003). Cada factor, en este sentido, representa la relación existente entre un conjunto de variables intercorrelacionadas, y explica el máximo de su varianza común, es decir, que los factores pueden interpretarse como las dimensiones subyacentes de un conjunto amplio de variables.

A lo largo del trabajo, la premisa fue no introducir en el modelo datos previamente agrupados en la etapa de diseño de los sistemas clasificatorios, sino hacer uso de la información en su máximo nivel de desagregación y obtener su agrupamiento espacial como un resultado del análisis. Sin embargo, se pretendía incorporar como insumo del análisis factorial tanto indicadores simples de los fenómenos y

\footnotetext{
${ }^{6}$ La estructura espacial de la mortalidad constituyó una importante excepción, ya que para su estudio no se puede prescindir de las Estadísticas Vitales, que en Argentina se publican para grandes territorios (los Partidos del Gran Buenos Aires y las Comunas de la Ciudad Autónoma de Buenos Aires). Si bien existen metodologías para construir algunos indicadores básicos del nivel de la mortalidad con información censal (United Nations, 1983), los datos requeridos suelen tener serios problemas de calidad y el Censo 2001 no los publica para las unidades espaciales de estudio.

${ }^{7}$ Para más información acerca de las posibilidades y limitaciones para el análisis microespacial de los Censos Argentinos véase Mera y Marcos, 2012.
} 
las estructuras sociodemográficas, como sistemas clasificatorios complejos con numerosas categorías. En este último caso -y con el fin de equilibrar la cantidad de indicadores de cada fenómeno/ estructura introducida en el modelo final-, se aplicó un análisis factorial de primer grado para reducir la cantidad de variables, y luego los factores obtenidos se introdujeron junto a los indicadores más simples como insumo de un análisis factorial de segundo grado, que brindó los resultados finales que se analizan en el próximo apartado.

A continuación se listan las variables introducidas en el análisis factorial de segundo orden, pudiéndose consultar en el Anexo las variables originales que resumen los factores obtenidos en el análisis factorial de primer orden:

1. Nivel socioeconómico

1.1. Factor de nivel socioeconómico.

2. Estructura por edad y sexo

2.1. Relación de masculinidad

2.2. Edad media

3. Estructura de los hogares y las familias

3.1. Tamaño de los hogares multipersonales

3.2. Factor Familias con hijos en edad escolar vs. Hogares de personas activas sin hijos

3.3. Factor Familias con hijos pequeños vs. Parejas maduras sin hijos y Hogares no familiares de personas pasivas

4. Nupcialidad

4.1. Factor unidos vs. viudos y separados/divorciados

4.2. Factor casados vs solteros y separados/divorciados

4.3. Edad de la mujer a la unión7

4.4. Factor homogamia escolar perfecta (secundaria incompleta o más vs hasta primaria completa)

5. Fecundidad

5.1. Paridez media final

5.2. Edad media al primer hijo8

6. Migración 
6.1. Porcentaje de migrantes internos

6.2. Porcentaje de migrantes internacionales

6.3. Porcentaje de migrantes internos recientes

6.4. Porcentaje de migrantes internacionales recientes

\section{RESULTADOS}

Como resultado se obtuvieron tres factores con autovalor superior a 1, que se han rotado mediante el procedimiento ortogonal Varimax, y que en conjunto explican el 88,2\% de la heterogeneidad que imprimen en el espacio las dieciséis variables que se han integrado en este análisis factorial de segundo grado (Tabla 1).

Variables

Nivel socioeconómico

Relación de masculinidad

Edad media

H. no familiares - flias. completas

Tamaño de los hogares multipersonales

Flias. con hijos en edad escolar - H. de personas activas sin hijos

Flias. con hijos pequeños - Parejas maduras s/hijos y H. no fliares. de personas pasivas

Unidos vs. viudos y separados/divorciados

Casados vs solteros y separados/divorciados

Edad de la mujer a la unión

Homogamia escolar perfecta (secundaria incompleta o más vs hasta primaria completa)

Paridez media final

Edad media al primer hijo

Porcentaje de migrantes internos

Porcentaje de migrantes internacionales

Porcentaje de migrantes internos recientes

Porcentaje de migrantes internacionales recientes

Valor propio

$\%$ de la varianza

$\%$ de la varianza acumulada

Factor 1 Factor 2 Factor 3

$-0,968$

0,848

$-0,981$

0,746

$-0,509$

0,925

0,758

$-0,917$

$-0,970$

0,845

$-0,915$

0,911

0,958

$-0,967$

0,632

0,680

0,949

0,917

0,802

10,0

3,6

1,4

58,8

58,8

Tabla 1. Matriz factorial integradora (1), y varianza explicada.

Fuente: autoría propia.

Notas: (1) Método de extracción: Análisis de componentes principales. 
El Factor 1, con autovalor de 10 y capacidad explicativa del 58,8\%, es sin dudas el más significativo. De acuerdo a la matriz factorial (Tabla 1), satura en doce de las dieciséis variables: en el nivel socioeconómico de la población y en las variables de estructura y dinámica demográfica, con la excepción de las dos variables que se sabía con antelación que no estarían integradas (una de ciclo de vida familiar y otra de nupcialidad) 9 y de tres de las variables de migración. Es decir que este factor de gran capacidad explicativa identifica a grupos socioeconómicos contrapuestos y a su estructura y dinámica demográfica específica:

- De un lado, la población de nivel socioeconómico alto, que se asocia en el espacio a:

- la estructura por edad envejecida, que explica otras características de estas unidades espaciales, como la estructura por sexo feminizada y la presencia relativamente alta de viudos, la presencia de hogares de tipo no familiar de personas pasivas y de familias en la etapa nido vacío de su ciclo de vida, y el pequeño tamaño de los hogares multipersonales,

- la dinámica demográfica lenta, en la que las mujeres se unen tardíamente, posponen en el tiempo la reproducción y, cuando tienen hijos, son relativamente pocos,

○ y, finalmente, a signos de consolidación temprana de esos sectores de la ciudad como espacios residenciales de estos grupos -el porcentaje de migrantes internos es bajo- y de reproducción de esta característica a través de las pautas de formación de las parejas -la homogamia escolar indica que es frecuente que las parejas se conformen entre personas de nivel educativo elevado-;

- En el polo opuesto, la población de nivel socioeconómico bajo, que aparece asociada en el espacio a:

- la estructura por edad joven, que se vincula, a su vez, con otras características de estas unidades espaciales, como la estructura por sexo relativamente equilibrada, la presencia de familias completas (muchas en etapa de expansión), el mayor tamaño de los hogares multipersonales y la elevada proporción de unidos,

- la dinámica demográfica más acelerada, en la que las mujeres se unen temprano, tienen hijos siendo más jóvenes y forman familias más grandes, 
$\circ$ y, finalmente, a signos de consolidación reciente de esos sectores de la ciudad como espacios residenciales de estos grupos -el porcentaje de migrantes internos es alto- y de reproducción de esta característica a través de las pautas de formación de las parejas -la homogamia escolar indica que es frecuente que las parejas se conformen entre personas de nivel educativo bajo-.

Las relaciones halladas son muy fuertes y evidentes, y se encuentran avaladas por los antecedentes que dan cuenta de que la estructura y la dinámica demográfica son diferenciales por nivel socioeconómico. Atendiendo a ello -y sin gran perjuicio de que el resultado esté afectado por la llamada "falacia ecológica"-, se llamó a este factor Estructura sociodemográfica predominante, y expresa en su polo positivo a los Grupos de nivel socioeconómico alto, su estructura envejecida y feminizada y su dinámica demográfica lenta y en el polo negativo a los Grupos de nivel socioeconómico bajo, su estructura joven y equilibrada por sexo y su dinámica demográfica acelerada.

Las unidades espaciales de nivel socioeconómico alto y muy alto, estructura envejecida y desequilibrada por sexo, dinámica demográfica lenta y consolidadas tempranamente como parte de la ciudad se encuentran distribuidas en buena medida de forma contigua en el mapa, conformando un gran área homogénea que comprende los tercios central y norte de la CABA -con la importante excepción del centro administrativo y de negocios de la ciudad en el centro-este, que se ha degradado socialmente-, el eje litoral norte que se extiende sobre las unidades costeras de los Partidos de Vicente López y San Isidro, y las inmediaciones de los ejes del ferrocarril que dinamizaron el crecimiento de la ciudad hacia la periferia oeste y sur durante la etapa desarrollista (Figuras 1 y 2).

A esta zona central con características sociodemográficas bien definidas, sigue un conjunto de unidades espaciales de composición sociodemográfica heterogénea, identificadas en el mapa con amarillo, que funciona espacialmente como antesala a la amplia periferia de nivel socioeconómico bajo y muy bajo, estructura joven y equilibrada por sexo, dinámica demográfica más acelerada, y consolidada como parte de la ciudad hace relativamente poco -ello queda evidenciado en la presencia alta de migrantes 
internos y además concuerda con los antecedentes acerca de la expansión física de la ciudad-. Estas últimas características se acentúan más en las proximidades de los bordes externos de la Aglomeración y en los espacios intersticiales, es decir, en las zonas identificadas con color rojo desde las que el centro es menos accesible, ya sea porque se encuentran a mayor distancia con respecto a él, o porque se encuentran fuera del área de influencia del medio transporte principal que conducía a él en la etapa desarrollista el ferrocarril . Algunas unidades espaciales este último grupo aparecen localizadas en zonas de composición opuesta fragmentándolas. Ellas corresponden a villas de emergencia y asentamientos informales en los que las familias de menores recursos encuentran una alternativa para acceder a las zonas próximas al centro y las fuentes de trabajo. 




Figura 2. Unidades espaciales de estudio: distribución según la puntuación obtenida en el Factor Estructura sociodemográfica predominante. AGBA, 2001.

Fuente: elaboración personal en base a INDEC. Censo Nacional de Población, Hogares y Viviendas 2001.

En líneas generales, este primer factor expresa un patrón sociodemográfico axial con centro en las inmediaciones del centro administrativo y de negocios, y ejes que se despliegan desde allí hacia las afueras de la ciudad, coincidentes con el tendido ferroviario. En la constitución de este rasgo han sido 
fundamentales: la inercia ejercida por el centro sinónimo de concentración de valiosos recursos como oportunidades laborales, equipamientos urbanos y servicios en general y viviendas de calidad a lo largo de la historia de la Aglomeración; la localización de los establecimientos productivos manufactureros durante la etapa de acumulación dinamizada por la expansión industrial, que buscaron aprovechar las ventajas locacionales que ofrecía la proximidad a la Ciudad Autónoma de Buenos Aires; las oportunidades habitacionales que se generaron mediante políticas de transporte y de tierras; y las estrategias residenciales desplegadas por las familias para satisfacer sus necesidades habitacionales de manera acorde a sus expectativas y en función de sus posibilidades.

El Factor 2 explica otro 21\% de la variabilidad que introducen las características sociodemográficas de la población en la ciudad, y resume en buena medida la distribución espacial de la Estructura de los hogares. Su polo positivo se asocia a unidades espaciales en las que los Hogares no familiares tienen alta representación (Tabla 1). Otras características completan y precisan el panorama en las unidades espaciales reunidas en este polo: cuando hay familias, suelen estar recién conformadas (son parejas de personas activas sin hijos), y tienen más presencia que en otras partes de la ciudad los solteros, separados y divorciados, los migrantes internacionales recientes y los migrantes internos. En el polo negativo están representadas las Familias completas y las Familias con hijos en edad escolar en general. Estas mismas unidades espaciales presentan además otros rasgos relacionados con el tipo de hogares que predomina en ellas: son frecuentes las personas casadas, y a la vez son muy pocos los migrantes (cualquiera sea la procedencia que se considere), es decir, las personas que presumiblemente están en proceso de asentarse y no han formado su familia en la ciudad. En el caso de este segundo factor debe tenerse, sin embargo, cautela a la hora de interpretar los resultados, puesto que a pesar de la coherencia encontrada entre las características que reúne, no puede tenerse certeza absoluta acerca de si corresponden a una única población que las reúne o a poblaciones diferentes que coinciden en el espacio.

La distribución de la composición de los hogares que expresa el factor sigue en gran medida la forma de anillos concéntricos con núcleo en el centro-este de la ciudad. Los hogares no familiares tienen su espacio residencial privilegiado en unidades espaciales contiguas ubicadas en el centro-este de la 
Aglomeración, que abarcan el litoral central de la CABA, incluyendo el centro administrativo y de negocios. Dentro de esta zona, el núcleo en el que el patrón es muy definido se encuentra identificado en rojo en el mapa de la Figura 3, y reúne un pequeño grupo de unidades espaciales centro-litoraleñas. Lo rodea un anillo de unidades espaciales anaranjadas en el que los hogares no familiares se encuentran presentes, pero en menor medida. Luego, un segundo anillo amarillo de unidades espaciales de composición de los hogares indefinida representa la transición hacia un tercer amplio anillo celeste en el que predominan las familias. Este último anillo, se despliega abarcando el oeste y sudoeste de la CABA y gran parte de la primera corona de Partidos del Gran Buenos Aires, coincidiendo aproximadamente con la zona de emplazamiento de la mayor parte de los establecimientos productivos de tipo industrial en la etapa desarrollista. La periferia de la Aglomeración, constituye un cuarto anillo en el que la composición familiar no está muy definida, salvo por unidades espaciales aisladas en las que son frecuentes los migrantes y otras en las que queda de manifiesto la composición eminentemente familiar.

También en el caso de este factor que expresa la estructura de hogares puede "leerse" el uso residencial del suelo que hacen los hogares como cristalización de factores estructurantes que hegemonizaron la dinámica de producción socioespacial en distintos momentos: el centro, con sus viviendas más pequeñas y costosas, y acceso privilegiado a servicios, lugares de esparcimiento, trabajo y estudio, resulta atractivo para los hogares que conforman personas que viven solas o en el marco de arreglos residenciales no familiares o para familiares sin descendencia: jóvenes estudiantes 0 profesionales que salieron de su familia de origen pero no conformaron aún una propia, parejas en edad activa sin hijos y ancianos. La periferia cercana, con buena infraestructura urbana, viviendas más amplias, oferta de empleo en industrias y proximidad al centro de la CABA, parece ser muy buscada por las familias.

Por fuera de la primera corona de Partidos la ciudad industrial comienza a desdibujarse y el rasgo predominante es la heterogeneidad en la composición de los hogares, salvo algunas unidades espaciales puntuales que presentan otra característica. En estas áreas se superponen capas de espacio con distinta 
impronta, en la medida que en 2001 las viejas cabeceras de los municipios y su conurbación empezaban a convivir con los nuevos barrios cerrados y parques industriales.

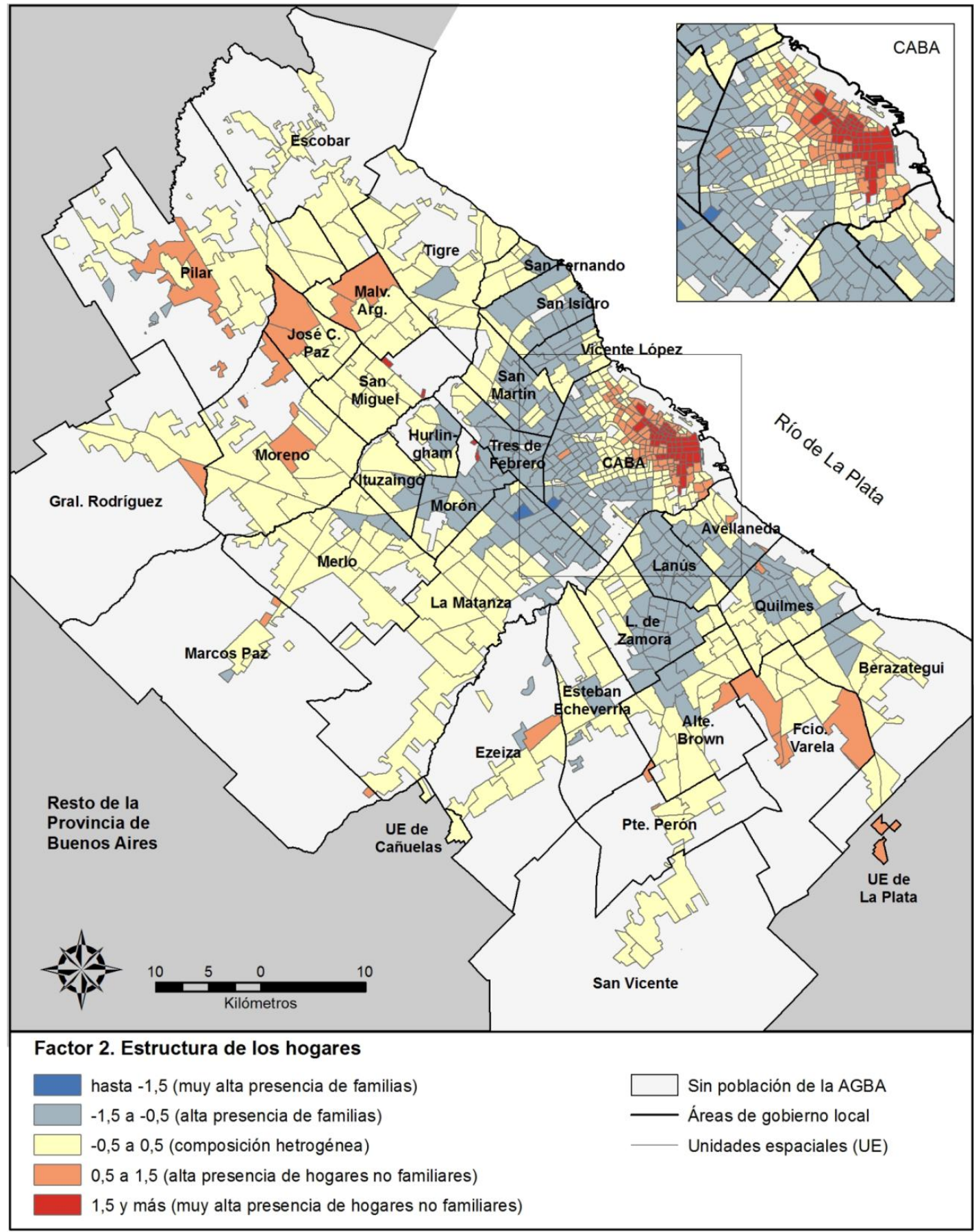

Figura 3. Unidades espaciales de estudio: distribución según la puntuación obtenida en el Factor Estructura de los hogares. AGBA, 2001.

Fuente: elaboración personal en base a INDEC. Censo Nacional de Población, Hogares y Viviendas 2001.

El tercer y último Factor que se ha retenido tiene autovalor apenas por encima de $1(1,4)$, explica el $8,4 \%$ de la varianza bastante menos que los dos factores anteriores (Tabla 1), y da cuenta del Componente migratorio de la ciudad. A pesar de la relativamente baja capacidad explicativa del factor 
y de que la migración ya no tenía en 2001 la relevancia que supo tener en el pasado, la Figura 4 revela que la concentración de los migrantes en determinadas unidades espaciales de la ciudad es muy fuerte y tiene un importante impacto local que merece, por lo tanto, ser analizado.

Interesan, fundamentalmente, las unidades espaciales con puntuaciones factoriales altas y muy altas, que aparecen identificadas en el mapa de en color anaranjado y rojo, respectivamente, y manifiestan la concentración de migrantes internacionales, que comparten su espacio residencial con los migrantes internos recientes (llegados entre 1996 y 2001, los cinco años anteriores al censo).

La mayor parte de estas relativamente pocas unidades espaciales se encuentra dentro de la CABA, más precisamente en el centro administrativo y de negocios y en los barrios pobres y villas de emergencia del sur de la Ciudad, y en algunas unidades espaciales de nivel socioeconómico alto. El conjunto de unidades espaciales se caracteriza por representar hábitats y condiciones de vida polares: de un lado el centro socialmente degradado y las villas miseria y barrios informales, y en el extremo opuesto zonas residenciales de hogares de elevado estatus. Pero al margen de estas diferencias, el centro y las zonas acomodadas tienen en común el hecho de concentrar la demanda de trabajadores no calificados, por sobre todo cadetes de distinto tipo y servicio doméstico y de limpieza, y las villas y los sectores céntricos degradados ofrecen la oportunidad de acceder a una vivienda en las inmediaciones de estas fuentes de trabajo, a costa de las condiciones de vida deficitarias que ofrecen. 


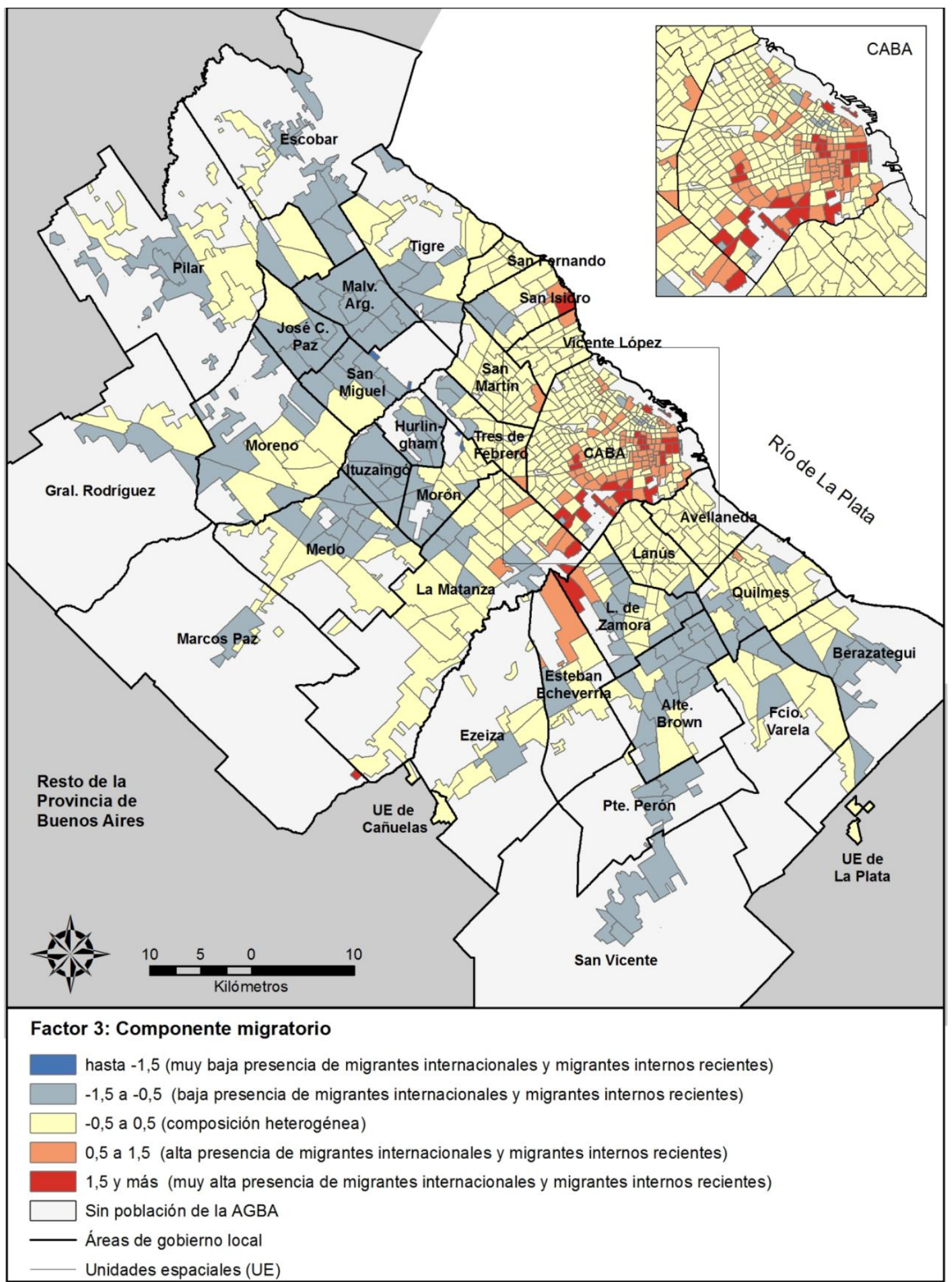

Figura 4. Unidades espaciales de estudio: distribución según la puntuación obtenida en el Factor Componente migratorio. AGBA, 2001.

Fuente: elaboración personal en base a INDEC. Censo Nacional de Población, Hogares y Viviendas 2001. 


\section{DISCUSIÓN Y CONCLUSIONES}

Como resultado de la investigación se identificaron en la Aglomeración Gran Buenos Aires tres grandes dimensiones espacialmente no relacionadas entre sí: la Estructura sociodemográfica predominante, que distingue a los grupos de nivel socioeconómico alto, con estructura envejecida y feminizada y dinámica demográfica lenta, de los grupos de nivel socioeconómico bajo, con estructura relativamente joven y equilibrada por sexo y dinámica demográfica acelerada; la Estructura de los hogares, que identifica por un lado el espacio residencial privilegiado de los Hogares no familiares y las parejas sin hijos y, por otro, el de las Familias completas y en general las familias que tiene en su composición niños en edad escolar; y, finalmente, el Componente migratorio, que da cuenta de la concentración de los migrantes sectores de la ciudad bien específicos (Figura 6). A través del recorrido exploratorio realizado hasta aquí se ha arribado de forma inductiva a lo que los ecólogos urbanos proponían entre las décadas de 1960 y 1970 como "tres dimensiones independientes del espacio social" (Murdie, 1969, citado en Bourgoignie, 1976), haciendo alusión al hecho de que las características sociodemográficas de la población de las ciudades se agrupan en tres grandes componentes o dimensiones independientes unas de otras, que varían espacialmente según tres configuraciones diferentes: "la variación axial del estatuto socioeconómico (modelo sectorial), la variación concéntrica de la estructura familiar y la segregación localizada de los grupos étnicos particulares cuyas variaciones no se conforman exactamente ni al esquema concéntrico, ni al esquema axial" (Bourgoignie, 1976: 200-201). Gráficamente, el modelo era representado por Bourgoignie inspirado en Murdie como muestra la Figura 5.

Sin embargo, el modelo de Murdie se basa en la morfología de las ciudades norteamericanas, que presentan marcadas diferencias con respecto a las latinoamericanas. Es decir que si se lo compara con los hallazgos de esta investigación (Figura 6) se pueden señalar similitudes, pero también divergencias. 
Las importantísimas similitudes son tres aspectos bien generales ya mencionados: la cantidad de dimensiones identificadas, las características sociodemográficas que reúne cada una de ellas y su configuración espacial interna.

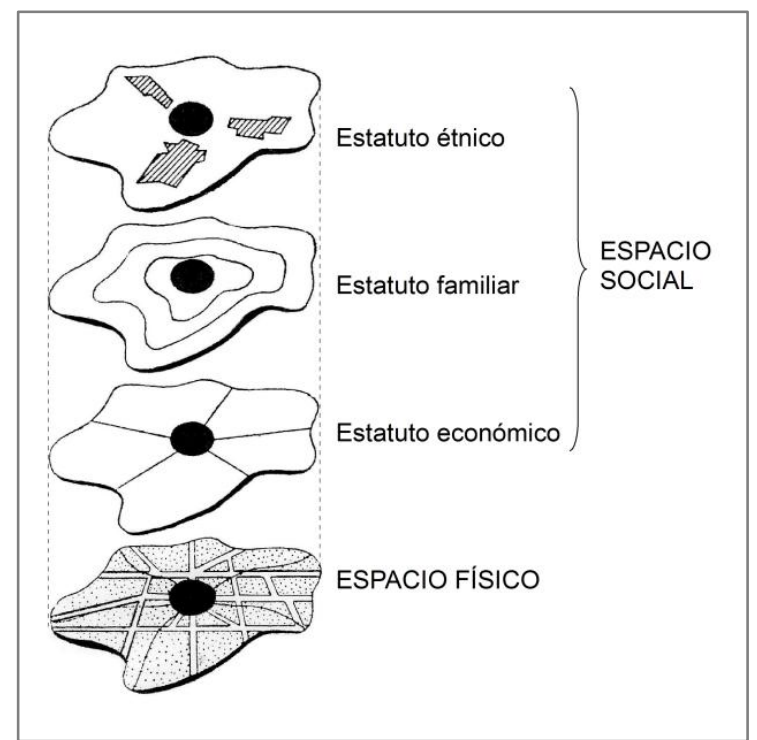

Figura 5. Modelo de las estructuras espaciales urbanas de Robert Murdie (1969).

Fuente: Bourgoignie, 1976: 199.

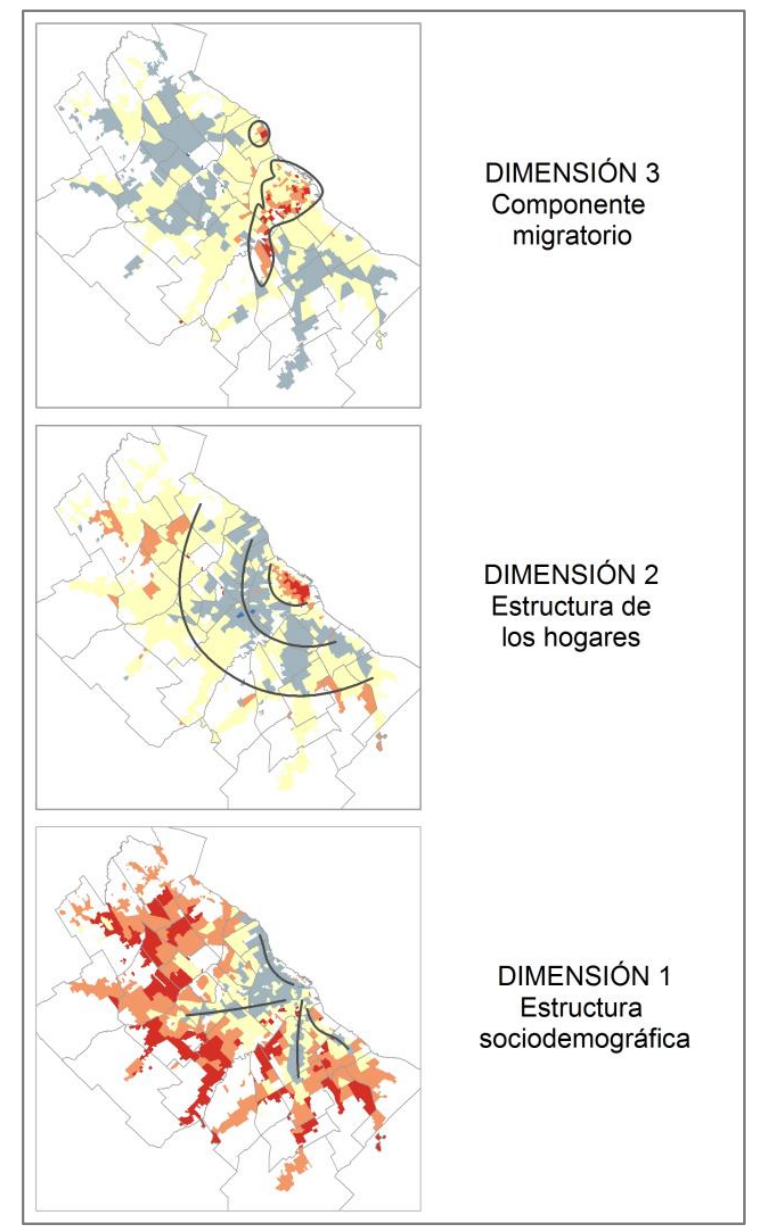

Figura 6. Síntesis de la estructura socioespacial de la AGBA en el año 2001

Fuente: elaboración personal en base a INDEC. Censo Nacional de Población, Hogares y Viviendas 2001.

Ahora bien, si se dejan a un lado las generalidades y se focaliza en un nivel de mayor detalle, se visualizan diferencias también importantes, particularmente entre la configuración espacial de lo que aquí se ha denominado "Estructura sociodemográfica predominante" y la del Estatuto económico del modelo. Las diferencias remiten a los patrones de asentamiento distintos que siguen los estratos sociales en las ciudades norteamericanas y latinoamericanas: de modo esquemático, en Norteamérica las clases altas privilegian el acceso al entorno "abierto" de la periferia y los sectores populares el acceso a las zonas industriales y las vías de transporte que conducen radialmente hacia el centro; $\mathrm{y}$ en Latinoamérica el esquema se invierte, y los sectores mejor posicionados en la estructura social 
ocupan las inmediaciones del centro y las zonas mejor conectadas con él y con las principales fuentes de trabajo en general, donde la dotación de servicios urbanos es mayor y de mejor calidad, y las clases bajas se distribuyen en zonas degradadas próximas al centro y en la amplia periferia. La suburbanización de las elites latinoamericanas constituye un elemento novedoso y relativamente reciente, que toma la forma de urbanizaciones cerradas que irrumpen en los amplios espacios socialmente degradados de la periferia, fragmentándolos.

Por otra parte, desde la ecología urbana se han hecho interpretaciones acerca del modelo de la Figura 5 que han sido objeto de no pocas críticas. En principio, la distinción propuesta en ese esquema entre el espacio físico y del espacio social en las ciudades, puede llevar a concluir que la dimensión física de la ciudad no es social. Sin embargo, el espacio físico urbano involucra tanto condiciones naturales como construcciones humanas, y éstas últimas son tan sociales como las desigualdades sociales de la población que se asienta en él, y su expresión socioeconómica (en condiciones de vida diferenciales), sociodemográfica (en estructuras y dinámicas demográficas distintas) y espacial (en el modo desigual en que los grupos se distribuyen en el espacio). Además, la ecología urbana otorga al medio físico un lugar entre los factores explicativos particularmente preminente, y con base en fuertes supuestos entre los que no faltan las analogías entre el mundo social y el natural, como los muy criticados planteos de selección y competencia entre los grupos, la adaptación funcional al medio externo -que incluye innovaciones en materia de transporte y comunicaciones- y la búsqueda de equilibrio atribuida al desarrollo social y a la relación entre población y medio en general (Gottdiener y Feagin, 1988).

En este trabajo, en cambio, se planteó desde el comienzo la necesidad de interpretar el espacio social en el marco general del complejo y multidimensional proceso de producción y reproducción social, del que es a la vez resultado y parte constitutiva, aunque sin perder de vista que la información que se presenta corresponde específicamente a la dimensión del espacio-producto. La estructura socioespacial a la que se ha arribado constituye la cristalización de dinámicas de producción y organización general (inclusive espacial) de la vida, que abarcan tanto lo que ocurre a nivel macrosocial (en relación directa con el espacio, la planificación urbana y la dinámica del mercado inmobiliario) como microsocial [el conjunto de prácticas que abarca las estrategias de vida de los hogares en general (Torrado, 1998), 
entre ellas las estrategias habitacionales y las estrategias de captación de recursos para llevarlas a cabo (Di Virgilio, 2003; 2007)].

El estudio de la dinámica social general que produce en su desenvolvimiento la estructura socioespacial hallada excede a los objetivos de este artículo. Sin embargo, a lo largo del trabajo se ha enriquecido el análisis de los resultados obtenidos relacionándolos con antecedentes que dan cuenta de factores macrosociales y microsociales espacioestructurantes que han operado en Buenos Aires a lo largo de su historia.

Por otro lado, desde una perspectiva microanalítica, se podría objetar que el problema de la separación o concentración de los grupos en el espacio urbano -que las metodologías cuantitativas se limitan a concebir en su carácter físico- debe ser pensado como una cuestión vinculada a la experiencia y la interacción, en cuyo marco las diferencias y distancias se construyen como tales (Mera y Marcos, 2012). Sin embargo, "la crítica microanalítica a los modelos macro sólo adquiere pertinencia cuando estos últimos han alcanzado previamente sus límites explicativos y heurísticos" (Otero y Pellegrino, 2004:23), situación que, dada la señalada vacancia en los estudios sociodemográficos integrales de perspectiva espacial sobre Buenos Aires, se encontraba hasta ahora lejos de haber sido alcanzada.

Además de cubrir ese vacío, los hallazgos contribuyen a plantear otros interrogantes y conforman un sólido punto de partida desde el que se puede continuar avanzando con mayor facilidad, sorteando los desafíos metodológicos que supone estudiar una ciudad como Buenos Aires.

Desde el punto de vista teórico, una de las principales inquietudes que quedan planteadas remite a la necesidad de (contar con datos y) espacializar la estructura socio-ocupacional de la población y corroborar la correlación con la estructura sociodemográfica que se ha hallado aquí, indagando en otros factores que pudieran mediar en esa relación. Otra importante arista que despunta refiere a la articulación entre los espacios residenciales identificados, con claras fronteras socioespaciales de tipo físico, con otras fronteras de tipo simbólico, construidas en la interacción y la experiencia, que operan 
acotando los sectores de la ciudad y espacios de socialización que forman parte del espacio de vida de las personas y las familias.

Desde el punto de vista metodológico, se han sentado las bases para el estudio macrosocial y a la vez microespacial de la ciudad, proponiendo alternativas para el cálculo de indicadores de nivel socioeconómico y de estructura y dinámica demográfica basados en datos censales, ante la escases de información de otras fuentes para unidades espaciales pequeñas.

Y desde la perspectiva de las políticas públicas, los resultados presentados constituyen en sí importantes elementos para pensar, planificar y gestionar la ciudad en base a conocimientos sólidos acerca de su conformación socioespacial interna. A partir de qaquí quedan abiertas las puertas para la actualización regular de la información y el aprovechamiento de los avances que se realicen en la producción de datos microespaciales, y para las indagaciones en función de problemáticas específicas adaptando el área de estudio y la resolución espacial de análisis.

Ya sea desde el punto de vista teórico o desde la perspectiva de la gestión, el espacio constituye una dimensión de análisis esencial, y, hoy más que nunca, exige ser rescatada como tal por los estudios sociodemográficos que intentan contribuir a la comprensión de los procesos sociales, en tanto producto y condición de posibilidad de las relaciones que lo atraviesan.

\section{BIBLIOGRAFIA}

ABBA, A. P. Aspectos metodológicos e instrumentales. En ABBA, A. P., KULLOCK, D., NOVICK, A., PIERRO, N. y SCHWEITZER, M. Horacio Torres y los mapas sociales. La construcción teórica del caso de Buenos Aires. Buenos Aires: Cuentahilos, 2011. 170p. p.81-94.

AZPIAZU, D.; MANZANELLI, P. \& SCHORR, M. Concentración y Extranjerización. La Argentina en la Posconvertibilidad. Buenos Aires: Capital Intelectual, 2011. 110p.

BASUALDO, E. Sistema politico y modelo de acumulación: Tres ensayos sobre la Argentina actual. Buenos Aires: Atuel, 2011. 220p.

BOURGoignie, G. E. CARDYN, L. J., DANDEnAUlT, G., \& MARTÍN, P. F. Perspectivas en ecología humana. Madrid: Instituto de Estudios de Administración Local, 1976. 318p. 
BRIANO, L.E., FRITZSCHE, F. J y VIO, M. L. El lugar de la industria: Los parques industriales en la reestructuración productiva y territorial de la Región Metropolitana de Buenos Aires. EURE (Santiago), Santiago de Chile, v.29, n.86, p.109$135,2003$.

BUZAI, G. D. Mapas sociales urbanos. Buenos Aires: Lugar editorial, 2003. 384p.

BUZAI, G. D. y MARCOS, M. The Social Map of Greater Buenos Aires as Empirical Evidence of Urban Models. Journal of Latin American Geography, v.11, p.67-78, 2012.

CICCOLELLA, P. Globalización y dualización en la Región Metropolitana de Buenos Aires. Grandes inversiones y reestructuración socioterritorial en los años noventa. EURE (Santiago), Santiago de Chile, v.25, n.76, p.5-27, 1999.

CICCOLELLA, P. Transformaciones recientes en las metrópolis latinoamericanas. En FERNANDEZ CASO, M. V. y GUREVICH, R. (coord.), Geografía. Nuevos temas, nuevas preguntas. Un temario para su enseñanza. Buenos Aires: Biblos, 2007.

DI VIRGILIO, M. M. Estrategias residenciales y redes habitacionales. El acceso a la vivienda de familias de bajos ingresos en el Área Metropolitana de Buenos Aires. En Congreso de la Latin American Studies Association, Dallas, 27 al 29 de Marzo, 2003.

DI VIRGILIO, M. M. Trayectorias residenciales y estrategias habitacionales de familias de sectores populares y medios en Buenos Aires. Tesis (Doctorado en Ciencias Sociales) - Facultad de Ciencias Sociales. Universidad de Buenos Aires, 2007.

DPOUyT . Lineamientos Estratégicos para la Región Metropolitana de Buenos Aires. La Plata: Dirección Provincial de Ordenamiento Urbano y Territorial. La Plata: Subsecretaría de Urbanismo y Vivienda, Ministerio de Infraestructura, Vivienda y Servicios Públicos, Gobierno de la Provincia de Buenos Aires, 2007. 407p.

GARCÍA DELGADO, D. y CHOJO ORTÍZ, I. Hacia un nuevo modelo de desarrollo. Transformación y reproducción en el posneoliberalismo. En GARCÍA DELGADO, D. y NOSETTO, L., El desarrollo en un contexto postneoliberal. Buenos Aires, CICCUS-FLACSO, 2006. 308p. p.39-69.

GERMANI, G. \& GRACIARENA, J. Estructura social de la Argentina. Análisis estadístico. Edición de 1987. Buenos Aires: Ediciones Solar, 1955.

GORELIK, A. Buenos Aires: el fin de la expansión. En PIREZ, P. (ed.). Buenos Aires, la formación del presente. Quito: Olacchi, 2009. 304p. p.267-284

GOTTDIENER, M. y FEAGIN J. R. El cambio de paradigmas en sociología urbana. Urban Affairs Quaterly, v. 24, n.2, p.163-187, 1988.

GROISMAN, F. y SUÁREZ, A. L. Residential Segregation in Greater Buenos Aires. En ROBERTS, B. R. y WILSON, R. H. Urban Segregation and Governance in the Americas. New York: Palgrave Macmillan, 2009. 231p. p.39-54.

HARVEY, D. Espacios de esperanza. Madrid: Akal, 2000. 328p.

INDEC. Censo Nacional de población, hogares y viviendas 2001. Base de datos REDATAM.

INDEC. Censo Nacional de población, hogares y viviendas 2010. Base de datos REDATAM.

JANOSCHKA, M. El nuevo modelo de la ciudad latinoamericana: fragmentación y privatización. EURE (Santiago), Santiago de Chile, v.28, n.85, p.11-20, 2002. 
LATTES, A. Esplendor y ocaso de las migraciones internas. En TORRADO, S. (comp.). Población y Bienestar en Argentina del Primero al Segundo Centenario. Una historia social del siglo XX. Buenos Aires: EDHASA, 2007. 2 tomos. Tomo II, p.11-49.

LEFEBVRE, H. La revolución urbana. Madrid: Alianza Editorial, 1972. 198p.

LIVI BACCI, M. Introducción a la demografía. Ed. 2011. Barcelona: Ariel, 1993. 475p.

MAICHTRY, N. (2007). Emergencia y mutaciones del sistema urbano. En TORRADO, S. (comp.). Población y Bienestar en Argentina del Primero al Segundo Centenario. Una historia social del siglo XX. Buenos Aires: EDHASA, 2007. 2 tomos. Tomo II.p.47-84.

MARCOS, M. (2011). Base cartográfica para el estudio de diferencias intraurbanas en la Aglomeración Gran Buenos Aires: procedimientos técnicos para su realización. En GEOSIG, Luján, n.3, 2011, p.1-21.

MERA, G. y MARCOS, M. La dimensión espacial de las diferencias sociales. Alcances y limitaciones metodológicoconceptuales de las herramientas estadísticas para abordar la distribución espacial intraurbana. Revista Universitaria de Geografía (RUG), Bahía Blanca, v.20, p.41-66, 2012.

MORALES VALLEJO, P. El Análisis Factorial en la construcción e interpretación de tests, escalas y cuestionarios. Madrid: Universidad Pontificia Comillas, 2011.46p.

MORENO JIMÉNEZ, A. La estructura urbana en la periferia de Madrid: Carabanchel. Madrid: Departamento de Geografía. Universidad Autónoma de Madrid, 1986. 112p.

OTERO, H. y PELlEGRINO, A. Compartir la ciudad. Patrones de residencia e integración de inmigrantes en Buenos Aires y Montevideo durante la inmigración masiva. En OTERO, H. (dir.), El mosaico argentino. Modelos y representaciones del espacio y de la población, siglos XIX-XX. Buenos Aires: Siglo XXI Editores, 2004. 557p. p.19-69

PREVOT SCHAPIRA, M. F. (2002). Buenos Aires en los años 90: metropolización y desigualdades. EURE (Santiago), Santiago de Chile, v.28, n.85, p.31-50, 2002.

ROBIROSA, M. Micro-espacios, municipios y otras jurisdicciones locales. Problemas y recomendaciones para su tratamiento. En INDEC, Aspectos teóricos y metodológicos relativos al diseño conceptual de la cédula censal. Buenos Aires: Publicaciones del INDEC, 1996. Serie D n² 2 , tomo 2.

ROFMAN, A. B. Desarrollo regional y exclusión social. Transformaciones y crisis en la Argentina contemporánea. Buenos Aires: Amorrortu Editores, 1999. 373p.

ROFMAN, A. B. Las transformaciones regionales. En SURIANO, J. (coord.), Dictadura y democracia (1976-2001). Nueva Historia Argentina. Buenos Aires: Sudamericana, 2005. 557p. p.331-376.

SABATINI, F. La segregación social del espacio en las ciudades de América Latina. En BID, Desarrollo Social. Washigton DC: Documento de Estrategia, 2003. Disponible en http://www.iadb.org/sds/doc/SOCSabatiniSegregacion.pdf

SANTOS PRECIADO, J.M. La técnica factorial y sus aplicaciones en el campo geográfico. En Espacio, Tiempo y Forma, 1991, p.13-70.

SOJA, E. Postmodern Geographies: The Reassertion of Space in Critical Social Theory. London: Verso Press, 1989

THUILLER, G. (2005). El impacto socio-espacial de las urbanizaciones cerradas: el caso de la Región Metropolitana de Buenos Aires. EURE (Santiago), Santiago de Chile, v.31, n.93, p.5-20, 2005.

TORRADO, S. Estructura social de la Argentina: 1945-1983. Buenos Aires: Ediciones de la Flor, 1992. 556p. 
TORRADO, S. Vivir apurado para morirse joven. Reflexiones sobre la transferencia intergeneracional de la pobreza. Revista Sociedad, Buenos Aires, n. 7, 1995.

TORRADO, S. Historia de la familia en la Argentina moderna (1870-2000). Buenos Aires: Ediciones de la Flor, 2003. $701 \mathrm{p}$.

TORRADO, S. Modelos de acumulación, regímenes de gobierno y estructura social. En TORRADO, S. (comp.), El costo social del ajuste. (Argentina, 1976-2002). Buenos Aires: EDHASA, 2010. 2 tomos. Tomo I. p.21-62.

TORRES, Horacio. Evolución de los procesos de estructuración espacial urbana. El caso de Buenos Aires. Desarrollo Económico, Buenos Aires, 1975, p. 281-306.

TORRES, H. A. El mapa social de Buenos Aires en 1943, 1947 y 1960. Buenos Aires los modelos urbanos. Desarrollo económico, Buenos Aires, 1978, p. 163-204.

TORRES, H. A. El mapa social de Buenos Aires (1940-1990). Serie Difusión, 3. Buenos Aires: FADU- UBA, 1993.

TORRES, H.A. Cambios socioterritoriales en Buenos Aires durante la década de 1990. EURE (Santiago), Santiago de Chile, v.27, n.80, p. 33-56, 2001.

UNITED NATIONS. Manual X. Indirect techniques for demographic estimation. Population Studies, 81. New York : United Nations, 1983.

VAPÑARSKY, C. A. Primacía y macrocefalia en la Argentina: la transformación del sistema de asentamiento humano desde 1950. Desarrollo económico, Buenos Aires, p. 227-254, 1995.

VAPÑARSKY, C.A. La aglomeración Gran Buenos Aires: Expansión espacial y crecimiento demográfico entre 1869 y 1991. Buenos Aires: EUDEBA, 2000. 240p.

VARESI, G. La argentina posconvertibilidad: modelo de acumulación. Problemas del Desarrollo. Revista Latinoamericana de Economía, México, v.41, n.161, abril-junio de 2010.

\section{Anexo: Variables insumo del análisis factorial de primer grado}

\section{Nivel socioeconómico}

1. Dimensión hábitat

1.1. Porcentaje de población en hogares hacinados (más de 2 personas por cuarto)

1.2. Porcentaje de población en hogares con viviendas de CALMAT I

1.3. Porcentaje de población en hogares con viviendas de CALMAT II

1.4. Porcentaje de población en hogares con viviendas de CALMAT III, IV o V

1.5. Porcentaje de población en hogares con inodoro con descarga y desagüe a red pública

1.6. Porcentaje de población en hogares con inodoro con descarga y desagüe a cámara séptica 
1.7. Porcentaje de población en hogares con inodoro con descarga y desagüe a pozo ciego, con inodoro sin descarga o sin inodoro

2. Dimensión educación

2.1. Porcentaje de población de 6 a 17 años no escolarizada

2.2. Porcentaje de población de 25 a 44 años que asistió, pero ya no asiste, con máximo nivel educativo alcanzado Primario incompleto

2.3. Porcentaje de población de 25 a 44 años que asistió, pero ya no asiste, con máximo nivel educativo alcanzado Primario completo-Secundario incompleto

2.4. Porcentaje de población de 25 a 44 años que asistió, pero ya no asiste, con máximo nivel educativo alcanzado Secundario completo-Terciario/ universitario incompleto

2.5. Porcentaje de población de 25 a 44 años que asistió, pero ya no asiste, con máximo nivel educativo alcanzado Terciario/ universitario completo

3. Dimensión condición socio-ocupacional

3.1. Porcentaje de población ocupada en ocupaciones de calificación profesional

3.2. Porcentaje de población ocupada en ocupaciones de calificación técnica

3.3. Porcentaje de población en ocupada en ocupaciones de calificación operativa

3.4. Porcentaje de población ocupada en ocupaciones no calificadas

4. Dimensión pobreza

4.1. Porcentaje de población en hogares sin privaciones

4.2. Porcentaje de población en hogares con privación de recursos corrientes

4.3. Porcentaje de población en hogares con privación patrimonial

4.4. Porcentaje de población en hogares con privación convergente

5. Dimensión seguridad social

5.1. Porcentaje de ocupados sin aportes jubilatorios

5.2. Porcentaje de población sin cobertura médica

2. Ciclo de vida familiar y estructura de los hogares no familiares: Intenta dar cuenta de las relaciones de dependencia en el interior de una familia pero también en los hogares de tipo no familiar, 
para conocer el stock de hogares en cada categoría y a sabiendas de que las personas no se asocian entre sí para realizar un recorrido lineal por estos estadios.

1. Ciclo de vida familiar

1.1. Pareja en edad reproductiva sin hijos: familia nuclear sin hijos (incluye familias extensas y compuestas) en la que la mujer de la pareja es menor de 45 años.

1.2. Nido lleno: familia completa con hijos o monoparental (incluye familias extensas y compuestas).

1.2.1. Nido lleno I: familia completa con hijos o monoparental (incluye familias extensas y compuestas) en la que al menos uno de los hijos es menor de 6 años

1.2.2. Nido lleno II: familia completa con hijos o monoparental (incluye familias extensas y compuestas) en la que todos los hijos tienen 6 años o más y son económicamente inactivos.

1.2.3. Nido lleno III: familia completa con hijos o monoparental (incluye familias extensas y compuestas) en la que todos los hijos tienen 6 años o más y al menos uno es económicamente activo.

1.3. Nido vacio: familia nuclear sin hijos (incluye familias extensas y compuestas) en la que la mujer de la pareja tiene 45 años o más.

2. Hogares no familiares (unipersonales y no conyugales)

2.1. Hogares no familiares $I$ : hogares unipersonales y no conyugales compuestos por personas de hasta 40 años

2.2. Hogares no familiares II: hogares unipersonales y no conyugales compuestos por al menos una persona de 40 años y más económicamente activa

2.3. Hogares no familiares III: hogares unipersonales y no conyugales compuestos por personas inactivas, al menos una de las cuales tiene 40 años y más

\section{Situación conyugal}

- Soltero

- Unido

- Casado 
- Viudo

- Separado/divorciado

\section{Homogamia escolar perfecta}

- parejas con ambos cónyuges con nivel educativo hasta primaria incompleta

- parejas con ambos cónyuges con nivel educativo primaria completa/ secundario incompleto

- parejas con ambos cónyuges con nivel educativo secundario completo/ terciario o universitario incompleto

- parejas con ambos cónyuges con nivel educativo terciario o universitario completo 